\title{
PROBLEMAS DE EQUIDAD ASOCIADOS CON EL CAMBIO DE LAS PRUEBAS DE ADMISIÓN UNIVERSITARIA EN CHILE ${ }^{1}$
}

\author{
Mladen Koljatic M. y Mónica Silva R.
}

\begin{abstract}
En este trabajo se examina el proceso de reemplazo de la PAA por la PSU, advirtiéndose que esta última fue elaborada en un período demasiado breve para su debido desarrollo y validación. Por otro lado, la evidencia indica que, contrariamente a lo planteado por quienes promovían su implantación, las nuevas pruebas no se han traducido en mayor equidad en el ingreso a la universidad. Ello se explicaría porque los estudiantes provenientes de los sectores más desventajados no han contado con la preparación necesaria para responder a los mayores niveles de dificultad de las nuevas pruebas de admisión.
\end{abstract}

MLAdEN KolJatic. Ingeniero comercial de la Universidad Católica de Chile, MBA de la University of Michigan y doctor en educación (Ed.D.), con mención en educación superior, de Indiana University. Es profesor titular de la Escuela de Administración de la P. Universidad Católica de Chile.

Mónica Silva. Psicóloga de la P. Universidad Católica de Chile, Master of Science y Ph.D., con mención en psicología de la educación, de Indiana University. Es investigadora de la Escuela de Administración de la P. Universidad Católica de Chile.

${ }^{1}$ El presente trabajo es una adaptación y actualización del artículo "Equity Issues Associated with the Change of College Admission Tests in Chile” (2006), publicado en Equal Opportunities International, volumen 25, $\mathrm{N}^{\circ} 7$, pp. 544-567. Una versión previa fue presentada en el National Council for Measurement in Education Conference en 2003. 
ـ as naciones en desarrollo que emplean pruebas nacionales en sus procesos de admisión a la universidad tienen que ser particularmente rigurosas en su validación por las serias consecuencias asociadas a sus resultados tanto a nivel individual como de la sociedad en su conjunto. Mientras que en los países desarrollados una persona con educación universitaria gana aproximadamente 1,8 veces lo que gana una persona con estudios de educación básica, en Chile esa relación es de 5,5 veces (Beyer, 2000). Por su importancia en la movilidad social, un sistema bien planificado y justo de admisión a la universidad constituye en esencia un bien público, en la medida que otorga igualdad de oportunidades a todos los miembros de la sociedad (Heyneman, 2003).

En los países desarrollados, como por ejemplo en los Estados Unidos, se han establecido estándares para la elaboración de estas pruebas. Con ellos se busca garantizar que estén bien construidas y así proteger los derechos de todos quienes pudiesen verse afectados por ellas (American Educational Research Association [AERA], American Psychological Association [APA] y National Council on Measurement in Education [NCME], 1999). Sin embargo, en las naciones en desarrollo se presta menos atención a estos temas y a las consecuencias de las pruebas, no existiendo normas explícitas que fomenten las buenas prácticas (Chakwera, Khembo y Sireci, 2004).

El propósito de este trabajo es documentar el proceso de cambio de las pruebas de admisión en nuestro país, centrando la atención en temas de equidad, particularmente en las consecuencias que el abrupto cambio tuvo para los postulantes, especialmente para aquellos provenientes de colegios municipalizados. Del estudio se desprenden lecciones que pueden ser útiles para quienes están encargados de formular políticas públicas en pro de la equidad, en lo que atañe a la necesidad de cumplir con rigurosos estándares de evaluación para el diseño e implementación de pruebas de altas consecuencias.

\section{Enfoque de la investigación}

El proceso de cambio en las pruebas nacionales de admisión en Chile se aborda en estas páginas como un estudio de casos, aplicándose un enfoque cualitativo (Merriam, 1998). El estudio se basa, principalmente, en información de los medios de comunicación, datos de archivos y entrevistas con algunos de los participantes en el proceso. El período comprendido 
en este estudio se extiende desde diciembre de 1999 hasta enero del 2007, y cubre los antecedentes de los cambios, la controversia pública que rodeó el proceso y las consecuencias de la aplicación prematura de las nuevas pruebas.

En la elaboración del trabajo hemos contado con una ventaja que a la vez es una limitante. La ventaja consiste en disponer - por nuestra participación, aunque periférica, en el debate sobre la modificación de las pruebas de ingreso a la educación superior - de una óptica privilegiada de los procesos y relaciones entre los actores y los eventos que describimos, los que pudiesen no ser fácilmente captados por un observador externo y desconectado del proceso. La limitación es el riesgo de un compromiso emocional que pueda sesgar la discusión de los temas. Con el objeto de validar los juicios e interpretaciones de los datos y eventos que acá se detallan, hemos hecho un esfuerzo por minimizar la posibilidad de ese sesgo a través de extensas consultas y comunicaciones con expertos locales y extranjeros que no participaron directamente en la controversia.

\section{El contexto de la admisión universitaria en Chile}

\subsection{El proceso de admisión universitaria centralizado}

Desde mediados de los años sesenta, Chile tuvo un sistema centralizado de admisión a las universidades tradicionales. A los postulantes se les ponderaba el promedio de notas de la educación media y sus puntajes en pruebas estandarizadas de selección múltiple de matemáticas y verbal (Prueba de Aptitud Académica, en adelante PAA). Éstas habían sido construidas tomando como modelo a las pruebas de ingreso a la universidad SAT utilizadas en EE.UU.

La PAA examinaba sólo contenidos básicos cubiertos durante los dos primeros años de enseñanza media. Unas pocas de las más prestigiosas carreras requerían a sus postulantes rendir pruebas adicionales (Pruebas de Conocimientos Específicos, en adelante PCE) similares a los exámenes de asignaturas SAT-II. Las PCE eran pruebas estandarizadas de selección múltiple que examinaban contenidos avanzados en matemáticas, ciencias y ciencias sociales.

La PAA y las PCE eran administradas una vez al año por el Departamento de Medición y Registros Educacionales (DEMRE), un centro que operaba al interior de la universidad pública más grande del país, la Universidad de Chile. EL DEMRE desarrollaba las pruebas de admisión, las administraba y asignaba los puntajes, los cuales sólo eran válidos para el año en 
que el postulante rendía las pruebas. El DEMRE también procesaba las postulaciones para todas las carreras ofrecidas por las universidades financiadas con fondos públicos. La Universidad de Chile cobraba a los postulantes una tarifa por el servicio.

En las tres décadas de funcionamiento del sistema se realizaron pocos estudios destinados a evaluar las propiedades psicométricas de las pruebas. El último informe técnico del sistema global, publicado en 1990, indicaba que la batería de pruebas de admisión mostraba una confiabilidad de 0,90 para las PAA de matemáticas y verbal, y confiabilidades un tanto más bajas para las PCE. La capacidad predictiva de los puntajes estaba en el rango de otras pruebas de admisión internacionales (Díaz, Himmel y Maltes, 1990).

\subsection{La reforma educacional de los 90 y la decisión de cambiar las pruebas de admisión}

A fines de los 90, se estaba implementando en Chile una vasta reforma educacional cuyo propósito era, entre otros, mejorar la calidad y la equidad de la enseñanza secundaria (Brunner, 2005). Financiada mediante un préstamo del Banco Mundial, la reforma a la educación media fue liderada y diseñada por investigadores de elite que habían sido entrenados en prestigiosas universidades europeas y americanas. Entre sus medidas clave figuraban cambios obligatorios en el currículum y la extensión de la jornada escolar, ya que la significativa ampliación de los contenidos mínimos a enseñar hacía necesario aumentar el tiempo de clases. Según informe del Banco Mundial (The World Bank, "Implementation Completion Report...”, 2001), se esperaba que para el año 2003 los colegios municipales públicos y el sistema privado subsidiado estarían funcionando en jornada completa.

En un principio, el gobierno enfrentó dificultades en la obtención de apoyo político para la reforma debido a la falta de una estrategia comunicacional y a la limitada participación y consulta de actores claves. Señalaba al respecto un observador externo, "la iniciativa... tomó a la mayoría de la gente por sorpresa, gatillando muchas reacciones [adversas]" (Delannoy, 2000, p. 25). Posteriormente, luego de las turbulencias iniciales, el nuevo marco curricular para la educación secundaria fue aprobado y el Ministerio de Educación debía evaluar los resultados de la reforma, conforme a lo establecido en la Ley Orgánica Constitucional de Educación (LOCE) de 1990: “[C]orresponderá al Ministerio de Educación diseñar los instrumentos que permitan el establecimiento de un sistema para la evaluación periódica, 
tanto de la enseñanza básica como de la media, del cumplimiento de los objetivos mínimos de esos niveles [para lo cual se tendrán que] efectuar pruebas de evaluación, a lo menos, al término de la educación básica y de la educación media”.

En los primeros meses del año 2000, y no habiéndose aún diseñado los instrumentos de evaluación contemplados en la LOCE, el Ministerio de Educación convocó a una Comisión - compuesta por funcionarios del Ministerio, académicos de universidades y educadores - para evaluar las pruebas de admisión a la universidad. En noviembre del mismo año, en informe interno entregado en consulta a la ministra de Educación, la Comisión ministerial recomienda sustituir la batería de pruebas en uso (PAA y PCE) por un nuevo conjunto de pruebas que servirían el doble propósito de seleccionar estudiantes para la educación superior y evaluar el nuevo currículum de enseñanza secundaria. Textualmente, el informe señalaba: "Se hace necesario un giro fundamental respecto a la visión sobre las funciones de las pruebas de admisión: las pruebas no pueden ser más consideradas como un problema solo de la educación superior. Tienen una doble función: de selección y evaluación formativa de la EM [enseñanza media]... la doble función que debieran adquirir las nuevas pruebas orienta y vertebra el conjunto de proposiciones de cambio de la Comisión” (p. 42). Asimismo, el informe sugería que en la elaboración de las pruebas participara activamente el Ministerio de Educación y su Unidad de Currículum y Evaluación, dado que una de las funciones de dicha unidad consiste en "llevar un seguimiento sistemático de la implementación del currículum en las salas de clases... [y recoger] evidencia también sistemática sobre las diferencias entre currículum prescrito y currículum implementado en las diversas realidades institucionales de la educación media del país” (Comisión Nuevo Currículum, “Informe...”, 2000, p. 47).

A continuación, en el mismo mes de noviembre de 2000, el Ministerio de Educación informa al Consejo de Rectores —entidad compuesta por los rectores de las universidades financiadas con fondos públicos y presidida por el/la ministro de Educación - sobre los cambios recomendados en las pruebas de admisión (“Consejo de Rectores Decide”, La Segunda, 2000, noviembre 24). El proyecto para la elaboración de las nuevas pruebas, denominadas Sistema de Ingreso a la Educación Superior (en adelante, SIES), se financiaría con dineros públicos a través del Fondo de Fomento al Desarrollo Científico y Tecnológico (FONDEF). El SIES sería dirigido por dos académicos de las principales universidades chilenas, uno de los cuales había sido miembro de la comisión del Ministerio que había propuesto el cambio del sistema de admisión. 
El Consejo de Rectores accede al cambio en la batería de admisión a la universidad, y, en octubre del 2001, funcionarios del Banco Mundial que supervisaban el préstamo para la reforma de la educación secundaria comunicaban en un informe interno que las pruebas para el ingreso al sistema universitario estaban siendo revisadas por el Ministerio de Educación con la colaboración del Consejo de Rectores. El informe del Banco Mundial señalaba incluso que las nuevas pruebas de admisión podrían transformarse, con el tiempo, en exámenes de salida de la educación media, sirviendo así como mecanismo para garantizar la sostenibilidad de la reforma y la evaluación de sus resultados (The World Bank, "Implementation Completion Report...”, 2001).

\section{Las nuevas pruebas: Proyecto SIES}

La nueva batería de admisión SIES - a ser desarrollada en el curso de dos años - consistía en cuatro pruebas de selección múltiple: matemáticas, lenguaje, ciencia (cubriendo biología, física y química) y ciencias sociales. A los postulantes a cualquiera de las universidades del Consejo de Rectores se les exigiría rendir todas las pruebas SIES, las que examinaban el $100 \%$ del extenso y recientemente reformado currículum nacional obligatorio de la enseñanza media. Por ejemplo, la prueba SIES de ciencias sociales abordaría contenidos en geografía, economía y cinco siglos de historia nacional, desde las civilizaciones pre-hispánicas hasta la historia contemporánea, incluyendo los cambios políticos, sociales, económicos y culturales en Chile entre 1970 y el presente; además de tres mil años de historia universal, abarcando las primeras expresiones culturales de la humanidad y las primeras civilizaciones clásicas hasta la historia contemporánea, incluyendo la Gran Depresión de los años 30 (Vial, 2001).

En conformidad con el espíritu de la reforma de los años 90 de mejoramiento de la calidad y la equidad de la enseñanza, las nuevas pruebas fueron presentadas como un avance en tal sentido. Por su conexión con el currículum, los proponentes de las pruebas afirmaban en abril de 2002 que, entre otros beneficios, la nueva batería SIES tenía un potencial mayor de equidad en el proceso de selección de alumnos que las pruebas en uso (PAA y PCE); que por alinear las nuevas pruebas con los currículum de primero a cuarto medio se mejoraría la calidad de la educación secundaria; y que el uso de métodos basados en teoría de respuesta al ítem (IRT) permitiría equiparar las pruebas a través de los años, incrementando así la eficiencia del sistema de admisión (Bravo et al., 2000; Bravo y Manzi, abril 2002). 


\subsection{La controversia pública sobre el SIES}

La reacción pública ante la noticia del cambio en las pruebas fue lenta, posiblemente debido a la falta de información pública con que se contaba. El apoyo al proyecto por parte del Consejo de Rectores, los beneficios que se prometieron para el sistema escolar secundario y la reputación de los líderes del equipo, unidos a las afirmaciones de que el proceso estaba siendo conducido de acuerdo con los más altos estándares internacionales y empleando la última tecnología, contribuyeron a justificar el proyecto ante la opinión pública.

Sin embargo, la lógica misma del proyecto SIES, particularmente la conveniencia de usar el mismo instrumento para evaluar la reforma a la enseñanza media y servir como prueba de admisión para la educación superior, y sus potenciales consecuencias sociales, concitaron la atención de los medios. Confrontados con un ambiente de creciente crítica, los líderes del SIES negaron a continuación, en mayo de 2002, los auténticos motivos detrás del cambio — esto es, garantizar la sustentabilidad de la reforma educacional y evaluar sus resultados- declarando que tal interpretación era un "malentendido de parte del público” (Manzi y Bravo, mayo 2002, p. E6). Y ninguno de los participantes en la comisión del Ministro de Educación que aconsejó el cambio de pruebas, ni quienes tuvieron acceso al informe del Banco Mundial hablaron públicamente para clarificarlos².

Las aprensiones con respecto a los nuevos exámenes de admisión a la universidad se intensificaron luego de la publicación de una muestra de ítems en la página web del proyecto SIES. Académicos y educadores detectaron serias fallas en los ítems de las nuevas pruebas de matemática, ciencias y lenguaje, y enviaron cartas a la prensa advirtiendo acerca de ello (Rodríguez, 2002; Valenzuela y Cousiño, 2002; Bustos, Galanti y Díaz, 2002; Navarro, 2002). Otras personas cuestionaron la validez de algunos ítems que parecían poco razonables a los ojos del público (García-Huidobro, 2002; Bortolaso, 2002). Hubo también quienes manifestaron preocupación por los extensos contenidos cubiertos por las nuevas pruebas, lo que representaba una amenaza a lo que consideraban una ya restringida libertad de educación en el país. Su temor era que ello se tradujera en un empobrecimiento del currículum, limitando la incorporación de asignaturas opcionales no examinadas en las pruebas como filosofía, lenguaje y las artes (Fontaine, 2002; Ibáñez, 2002; “Seminario Prueba”, en “Visión Universitaria PUC”, 2002, julio).

${ }^{2}$ No obstante, hubo quienes intuyeron la motivación detrás del cambio señalando que ya existía una evaluación a nivel nacional para medir resultados educacionales, las pruebas SIMCE (Labarca, 2002). 
Inicialmente, salvo contadas excepciones, los académicos miembros de las universidades públicas representadas en el Consejo de Rectores no criticaron abiertamente la iniciativa promovida por sus propios rectores. El canal para la expresión de las dudas y objeciones al SIES lo abrió el Centro de Estudios Públicos (CEP), una organización privada sin fines de lucro, no partidista, inspirada en los principios y valores de una sociedad libre, cuyo objetivo es el de proveer un foro destinado a promover la discusión nacional de temas públicos.

Los investigadores del CEP organizaron mesas redondas y grupos de discusión para analizar las nuevas pruebas y las implicancias de los cambios para el sistema educacional chileno y para los postulantes que las rendirían, sirviendo como catalizadores para un debate académico sobre el proyecto. Los reparos hacia el SIES apuntaban principalmente a tres áreas: los fundamentos para tan radical cambio en las pruebas y los supuestos beneficios para el sistema educacional, la calidad técnica de los nuevos exámenes que estaban siendo desarrollados y la omisión de un marco de validación para las nuevas pruebas.

\subsection{Los presuntos beneficios de las pruebas SIES}

Entre los supuestos beneficios del SIES se contaban el mejoramiento del sistema escolar y la mayor equidad en las oportunidades educacionales para estudiantes de bajos recursos, particularmente aquellos provenientes de colegios municipales públicos.

Para apoyar el cambio, quienes desarrollaban las pruebas emplearon los mismos argumentos que usó Atkinson (2001) para demandar la modificación del SAT en EE.UU.: afirmaron que las nuevas pruebas SIES beneficiarían al sistema escolar al fortalecer la conexión entre el currículum de enseñanza media y el proceso de selección universitaria. También citaron estudios realizados en la Universidad de California por Geisser y Studley (2001), en los que se comparaba al SAT-I y al SAT-2 como instrumentos de admisión, para sostener que el cambio no perjudicaría a los más pobres, y ofrecieron evidencia nacional para apoyar los argumentos en pro de la equidad de las nuevas pruebas (Bravo y Manzi, 2002). Sin embargo, su evidencia estaba basada en una equívoca comparación entre la PAA y las PCE con las pruebas SIMCE. La comparación era inapropiada puesto que comparaba el SIMCE —una prueba sin consecuencias individuales para los alumnoscon las pruebas de admisión a la universidad que eran de altas consecuencias y, por lo cual, los postulantes se preparaban para rendirla. Adicional- 
mente, la comparación era sesgada ya que se centraba en el desempeño del muy pequeño y autoseleccionado grupo de postulantes que tomaban tanto la PAA como las PCE, éstas últimas requeridas sólo para los cupos en las más prestigiosas universidades (Beyer, 2002).

Dada la sustancial desigualdad socioeconómica en el país y el persistente fracaso de los colegios públicos en entregar a los alumnos las oportunidades necesarias para aprender, la presunción de que las nuevas pruebas tendrían el potencial de aumentar la equidad del acceso a las universidades fue cuestionada por investigadores del CEP. A diferencia de los colegios particulares pagados, no todas las escuelas públicas municipales estaban funcionando con jornada escolar completa y, por lo tanto, no cubrían todos los contenidos prescritos por el extenso currículum recientemente reformado. Bajo esas condiciones, la decisión de cambiar las pruebas en tan corto tiempo fue considerada injustificadamente prematura, pues se corría el riesgo de aumentar la ya sustancial brecha de desempeño entre estudiantes de colegios municipalizados y privados (Eyzaguirre y Le Foulon, 2002).

Con todo, la ministra de Educación, algunos políticos y líderes del Colegio de Profesores se hicieron eco de las expectativas creadas por los líderes del proyecto SIES, en el sentido de que las nuevas pruebas serían más equitativas que las que estaban en uso ("Ministra Aylwin Valida”, Las Últimas Noticias, 2002b, junio 13; Brunner, 2002; “Colegio de Profesores”, El Mercurio, 2002, julio 4). Sumado a lo anterior, los líderes del proyecto SIES también sugirieron que un beneficio extra de las nuevas pruebas sería una menor necesidad de entrenamiento por la vía de preuniversitarios ya que la sola concentración en el trabajo escolar sería suficiente como estrategia para tener un buen desempeño en ellas ("Dan a Conocer”, El Mostrador.cl, 2002, abril 4; “SIES, un Desafío”, EducarChile.cl, 2002, abril 26).

\subsection{La calidad técnica de las nuevas pruebas}

El aumento de la brecha en materia de equidad no era la única fuente de preocupación para los detractores del SIES. La calidad técnica de las nuevas pruebas también era un motivo de preocupación y la controversia sobre el SIES alcanzó su clímax después que un informe firmado por un grupo de matemáticos y educadores altamente calificados, congregados por el CEP para analizar la nueva prueba de matemáticas, confirmó que ésta era 
gravemente deficiente y cuestionó la competencia del equipo SIES para producir un buen examen para el año 2003 (Friedman et al., 2002).

Este reporte establecía que una gran proporción de los ítems en la página web del SIES presentaba defectos en su construcción, particularmente los nuevos ítems de respuesta graduada, que consistían en preguntas que permitían asignar puntaje a respuestas parcialmente correctas. El problema detectado no era menor, ya que aproximadamente el $40 \%$ de los ítems de respuesta graduada en la prueba SIES de matemáticas podía ser contestado sin siquiera leer el enunciado del problema ${ }^{3}$. La recomendación de la comisión de matemáticas del CEP fue mantener las pruebas existentes de admisión a la universidad y agregar gradualmente ítems que incorporaran las nuevas áreas de contenidos especificadas en la reforma curricular de la enseñanza media, otorgando suficiente tiempo al sistema escolar para adaptarse a estos cambios.

El CEP también convocó comisiones de académicos para estudiar las pruebas de lenguaje, ciencias sociales y ciencias, y sus informes tampoco fueron favorables a las nuevas pruebas (Elmes et al., 2002; Hojman et al., 2002; Fermandois et al., 2002).

Los líderes del proyecto SIES desconocieron las fallas de sus pruebas. En el caso de los ítems defectuosos (de respuesta graduada), restaron importancia a las consecuencias en varios comunicados a la prensa (Dusaillant, 2002). La controversia en los diarios terminó cuando un alto funcionario del Ministerio de Educación finalmente reconoció que la falla en los ítems de respuesta graduada existía (“Ministerio de Educación”, Las Últimas Noticias 2002a, junio 13). De todas formas, tanto los funcionarios del Ministerio como quienes estaban desarrollando las pruebas, insistían en que las críticas eran exageradas e irresponsables, reclamando que era parte de una conspiración políticamente motivada destinada a desacreditar el proyecto SIES (Jalilie, 2002; Urzúa, 2002b; “Ministra Aylwin Valida”, Las Últimas Noticias, 2002b, junio 13).

\footnotetext{
${ }^{3}$ En los ítems de respuesta graduada quienes respondían las pruebas sabían que entre las opciones había una respuesta correcta y una parcialmente o "aproximadamente” correcta. Si esa información era usada adecuadamente, les permitía escoger la opción correcta para algunos ítems sin siquiera leer el enunciado del problema. Por ejemplo, las opciones para un ítem SIES de respuesta gradual decían: (a) 9 (b) 27 (c) 39 (d) entre 35 y 40, y (e) sobre 40. La respuesta correcta podía fácilmente adivinarse porque había sólo una opción que permitía otra respuesta “aproximadamente” correcta. Treinta y nueve era la única respuesta que está contenida en el rango 35-40. Esta simple heurística permitía al que respondía seleccionar la respuesta correcta sin ningún conocimiento adicional (Dusaillant, 2002).
} 


\subsection{La omisión de un marco de validación}

La omisión de un marco de validación para los nuevos exámenes y el inadecuado cronograma para la eliminación de las pruebas en uso fue también cuestionado (Sepúlveda, 2002; Parada, 2002). En algunas universidades del Consejo de Rectores, los académicos advirtieron a sus rectores que no era ético experimentar con la primera generación de postulantes, convirtiéndolos en conejillos de indias, y recomendaron que la eliminación de la batería existente de pruebas fuera pospuesta por al menos dos años, hasta que se hubiese recogido evidencia sobre los beneficios de las nuevas pruebas (“Académicos de la USACH”, La Segunda, 2002i, agosto 16; “U. de Chile Pone Cautela”, La Segunda, 2002d, junio 19). Sugirieron que durante ese período se les exigiese a todos los postulantes rendir ambas baterías de pruebas (PAA y SIES), de manera de evaluar el funcionamiento de éstas y conducir los estudios de validación omitidos por el SIES (Astorga, 2002; “Riveros Propone”, La Segunda, 2002c, julio 17; Urzúa, 2002a).

En vista de las muchas inquietudes sin respuesta canalizadas a través de la prensa y de la preocupación expresada en círculos académicos sobre los motivos de la urgencia en la sustitución de las pruebas, circularon rumores a fines de junio y principios de julio del 2002 de que la fecha para el debut de estos exámenes, planificado para diciembre del 2003, tendría que ser pospuesto (“Comisión de la U. de Chile”, La Segunda, 2002a, julio 4; “SIES la Presión”, La Segunda, 2002b, julio 4). No obstante, en medio de un clima de incertidumbre y rumores, el Consejo de Rectores decidió mantener el cronograma y esperar los resultados de una prueba de campo destinada a calibrar los ítems del SIES, programada para septiembre del 2002, antes de hacer un pronunciamiento final ("Rectores de 25 Universidades", La Segunda, 2002g, julio 25). Las declaraciones públicas de algunos rectores se hicieron eco de la noción errónea difundida por los líderes del proyecto SIES de que las consideraciones de validez sobre el uso de los puntajes de la prueba para fines de admisión quedarían ampliamente satisfechas después de la aplicación piloto para calibrar ítems (“El Sies Comenzará”, El Diario Austral, Valdivia, 2002, julio 21; Rosso, 2002a; Rosso 2002b).

Para agosto del 2002, la tensión se intensificó cuando dos influyentes rectores (de la Universidad de Chile y la Universidad de Santiago) decidieron que no adoptarían la nueva batería SIES para propósitos de admisión hasta que se pudiera proveer evidencia sobre los temas de validez pendientes. En cambio, requerirían a sus postulantes tomar las pruebas existentes junto a las nuevas (“Académicos de la USACH”, La Segunda, 2002i, agosto 16; “Comisión de la U. de Chile”, La Segunda, 2002a, julio 4). 
La ministra de Educación reaccionó públicamente con duras palabras acusando al rector de la Universidad de Chile de motivos pecuniarios tras su decisión. La administración de las pruebas de admisión era un negocio lucrativo al cual el rector de la Universidad de Chile estaba reticente a renunciar, de acuerdo a las expresiones de la máxima autoridad ministerial (“Guerra Declarada”, La Segunda, 2002j, agosto 26).

$\mathrm{El}$ incidente de los rectores disidentes fue percibido como una crisis que podía amenazar la continuidad del sistema centralizado de admisión al sistema universitario público ${ }^{4}$. Se llevó a cabo una reunión de emergencia de los rectores con la ministra de Educación para buscar una solución al problema SIES. El impasse fue resuelto con la decisión unánime del Consejo de Rectores de desarrollar un "examen de transición” ("Prueba de Admisión de Transición”, referida en la prensa como PAT), que más tarde fue rebautizada como Pruebas de Selección Universitaria (PSU).

La PSU representó una movida conciliatoria que intentaba acomodar algunas de las críticas al proyecto (“Transacción y Transición”, La Segun$d a, 2002 l$, agosto 30), pero sin dar el tiempo suficiente que permitiera conducir los estudios necesarios para garantizar una transición fluida entre los dos sistemas de admisión (Koljatic y Silva, 2002). La decisión del Consejo de Rectores de mantener el cronograma representó un apoyo tácito a la visión difundida por los líderes del SIES de que estudios adicionales de confiabilidad y validez para las formas finales eran un lujo que podía ser pasado por alto sin problemas ("Seminario Prueba", en "Visión Universitaria PUC”, 2002, julio 30). Así, las nuevas pruebas PSU serían desarrolladas en conjunto por el DEMRE y el equipo SIES en la Universidad de Chile, en el curso de un año.

\subsection{Concesiones}

Entre las concesiones hechas a los postulantes que rendirían las pruebas a fines del año 2003 estaba la reducción tanto del número de pruebas como de los contenidos a examinar. Se les exigiría tomar sólo tres de las

${ }^{4}$ Un poderoso estímulo para preservar una prueba de admisión a las universidades públicas única y centralizada, era que los fondos estatales estaban asignados a las instituciones que atraían a los estudiantes que obtenían los mejores puntajes en las pruebas. Esta política fue introducida a comienzos de los 80 por el Ministerio de Educación como una manera de estimular la competencia y la calidad de la educación entre las universidades. Aunque cualquier universidad representada en el Consejo de Rectores podía usar cualquier procedimiento de admisión para seleccionar estudiantes, el costo de no emplear las pruebas aprobadas era renunciar al acceso a estos recursos (Aravena y Molina, 2002). 
cuatro pruebas. Matemáticas y Lenguaje serían obligatorias, y la elección de la tercera prueba dependería de los requerimientos de las carreras a las cuales se quisiera postular. Una reducción de aproximadamente un $30 \%$ del contenido curricular fue sugerida para cada una de las pruebas y la decisión sobre las materias a omitir se dejó a un comité asesor compuesto por académicos (“Rectores Crean”, La Segunda, 2002h, julio 30). Sin embargo, la eliminación del contenido curricular sería temporal. Se agregarían anualmente materias, a objeto de que para el 2006 las pruebas de admisión incluyeran el $100 \%$ del currículum oficial cubierto en enseñanza media en las cuatro asignaturas, como lo especificaba el SIES.

Los contenidos a examinar en la primera versión de la PSU fueron revelados en noviembre del 2002 y se informó al público que facsímiles de las pruebas estarían disponibles en abril del 2003, ocho meses antes de su aplicación. Sin embargo, en abril del 2003 los postulantes fueron informados que sólo se entregarían muestras de ítems y no pruebas completas ("Escasez de Facsímiles”, La Segunda 2003b, abril 16; "Postulantes en Desventaja”, El Mercurio, 2003b, abril 17).

Las expectativas de que el trabajo escolar sería suficiente para obtener un buen desempeño, tal cual había sido sugerido por los líderes del SIES y funcionarios ministeriales (Herrera, 2001; "Dan a Conocer”, El Mostrador.cl, 2002, abril 14), tampoco se cumplieron. El cambio en las pruebas se tradujo en un próspero negocio para la industria de los preuniversitarios. Evidencia de lo anterior fue el comentario del gerente de un preuniversitario, de propiedad de una prestigiosa universidad del Consejo de Rectores, quien reconoció un aumento de un $30 \%$ de sus clientes para el 2003 ("El Ajuste”, El Mercurio, Suplementos Alternativas Académicas, 2003c, julio 8).

En marzo del 2003, el Consejo de Rectores informó sobre los requisitos de postulación a las carreras para el proceso anual de admisión. El peso asignado a las notas de enseñanza media aumentó dramáticamente en comparación con los años anteriores, lo que fue interpretado como un signo de desconfianza de las autoridades universitarias hacia la calidad de las pruebas que estaban siendo desarrolladas (“Académicos Detectan Recelos”, El Mercurio 2003a, marzo 28).

Durante el año, los líderes del proyecto SIES, los funcionarios del DEMRE, autoridades educacionales y rectores hicieron declaraciones tranquilizadoras en el sentido de que las nuevas pruebas PSU en desarrollo serían muy similares a las en uso y describieron el cambio como "menor" (“Postulantes Tendrán Menos”, La Segunda, 2003a, enero 23; "Pruebas de 
Selección”, El Mercurio, Suplemento Alternativas Académicas, 2003d, julio 8; "Puntajes de los nuevos exámenes", La Segunda, 2003c, julio 30; "Padres de la PSU”, La Segunda, 2003d, noviembre 2). Estas expresiones coincidían con la opinión de un ex consultor del Banco Mundial —asesor del Ministerio de Educación y del proyecto SIES - quien había manifestado en una entrevista que los cambios eran sólo una actualización tímida y cauta de pruebas anticuadas (Muñoz, 2002).

Finalmente, en septiembre del 2003, tres meses antes de la primera aplicación de la PSU, las autoridades anunciaron que se programaría para noviembre de ese año un ensayo gratuito de las pruebas de matemáticas y lenguaje ("En Noviembre”, El Mercurio, 2003e, septiembre 27) y se informó al mes siguiente que se ampliarían los tiempos de rendición de tres de las cuatro pruebas en 15 minutos (“Universidades Dan Más Tiempo”, El Mercurio, 2003f, octubre 7). Basándose en los resultados del ensayo, el rector de la Universidad de Chile anticipó que los puntajes de las nuevas pruebas serían más altos que en años previos. Estaba equivocado, pues hubo una significativa baja en los puntajes de las pruebas (“Alumnos Lograron”, $\mathrm{La}$ Segunda, 2003e, noviembre 28).

\section{Resultados de las primeras aplicaciones de la PSU}

Luego de la primera aplicación de la PSU en diciembre del 2003, funcionarios del Ministerio y altas autoridades educacionales se apresuraron en declarar que el proceso había sido un completo éxito, a pesar de que algunos resultados estaban lejos de ser satisfactorios, tanto para los postulantes como para las universidades. El número y composición de quienes rindieron la prueba cayó de 181.901 el año anterior a sólo 153.963, lo que representaba un $15 \%$ de pérdida, en su mayoría egresados de colegios municipales y postulantes de generaciones previas que no habían estado expuestos a la reforma curricular.

Hubo un mal funcionamiento de los ítems y problemas de escalamiento en dos de las nuevas pruebas. Las PSU de Matemáticas y Ciencias presentaban una alta asimetría en lugar de la distribución de campana deseable para pruebas de admisión. La dificultad promedio de los ítems en las pruebas de matemáticas y ciencia estaban en el rango de 0,30, muy lejos del valor esperado de 0,60 para pruebas con cinco opciones de respuesta (Henrysson, 1971). La marcada asimetría de las distribuciones de puntajes y el grado de dificultad de los ítems claramente indicaban un problema de mal 
ajuste entre el grado de dificultad de las pruebas y la distribución de las habilidades del grupo. Sin atender a ello, el comité técnico oficial para la PSU designado por el Consejo de Rectores, que incluía entre sus seis miembros a un funcionario del DEMRE y a los líderes del proyecto SIES, afirmó que desde un punto de vista estadístico el desempeño de las nuevas pruebas había sido altamente satisfactorio (Comité Técnico Asesor, 2004; Hawes, 2004).

Al poco tiempo de entregarse los puntajes de las pruebas en su primera aplicación, surgieron críticas en relación al escalamiento utilizado por el DEMRE. La normalización de los puntajes utilizada para transformar los puntajes en la PSU de Matemáticas fue cuestionada (Del Pino y Aravena, 2004). Sin embargo, ese cuestionamiento no reparó en que si los técnicos del DEMRE hubiesen usado el procedimiento sugerido por sus críticos, la brecha en rendimiento entre postulantes acomodados de colegios privados y los de colegios públicos municipales habría aumentado en un 8 por ciento. Ello habría sido impresentable, porque las nuevas pruebas habían

GRÁFICO N ${ }^{\circ}$ 1: TOTAL DE POSTULANTES QUE RINDIÓ LA PRUEBA POR TIPO DE ESTABLECIMIENTO

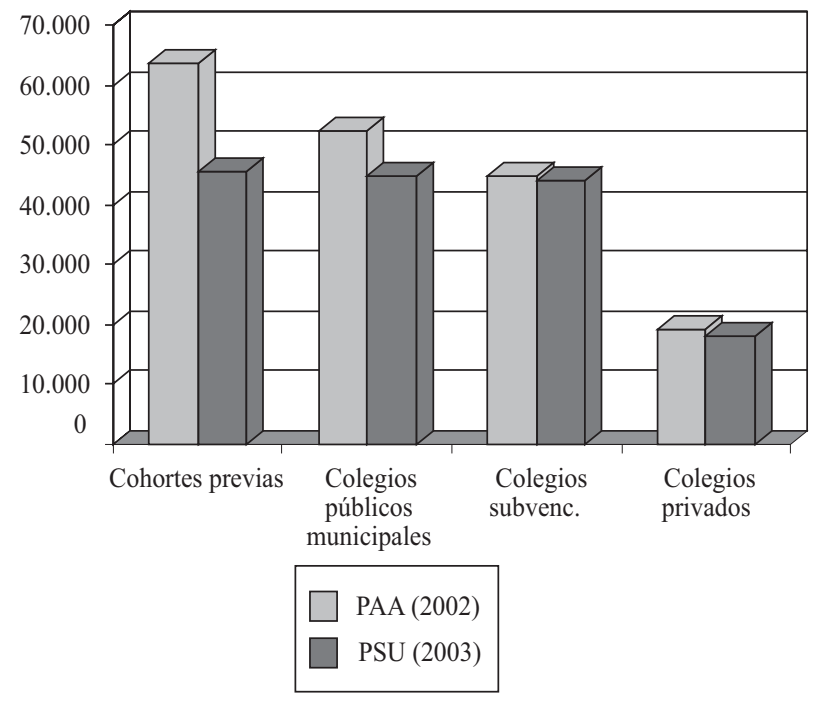

Fuente: Koljatic (2004). Datos entregados por D.A.R.A., P. Universidad Católica de Chile. 
sido promovidas como un medio para favorecer el acceso equitativo a la educación superior. El asunto de la magnitud de la brecha era en ese tiempo un tema que despertaba interés. Tanto así que el rector de la Universidad de Chile declaró a la prensa que la PSU había probado ser más equitativa para los estudiantes socioeconómicamente desfavorecidos ("Resultados de la PSU”, La Segunda, 2004a, enero 12), ignorando que la aparente reducción de la brecha era simplemente resultado de la transformación estadística usada por el DEMRE (Beyer, 2004).

Una consecuencia de la normalización empleada por el DEMRE en la PSU de Matemáticas fue una baja en los puntajes de las pruebas, que afectó principalmente al tramo superior de la distribución (ver Anexo). Los funcionarios de las universidades no fueron advertidos y el proceso de admisión continuó operando en base a los mismos puntajes de corte usados en el pasado. Como resultado del error, por primera vez en tres décadas no se llenaron cupos en prestigiosas carreras de universidades tradicionales. El descuido significó la migración de estudiantes calificados al sistema —más caro y menos prestigioso- de las universidades privadas ("Ues Tradicionales”, La Segunda, 2004b, enero 23). Asimismo, los puntajes de corte para las becas por méritos para estudiantes con necesidades económicas no fueron ajustadas a los cambios en la escala y, al menos en una prestigiosa universidad, no fueron íntegramente asignadas ese año (Delpiano, 2004).

En vez de reconocer los defectos del proceso, el Consejo de Rectores y los miembros de su comité PSU defendieron públicamente el cambio y afirmaron repetidamente que había funcionado de manera impecable. Sin embargo, los problemas fueron tácitamente reconocidos ya que unos pocos meses antes de la segunda aplicación de la PSU, en diciembre del 2004, el rector de la Universidad de Chile contrató los servicios del Educational Testing Service (ETS), organización norteamericana sin fines de lucro especialista en desarrollo de pruebas de admisión universitaria. Los expertos del ETS realizaron una auditoría de la PSU y emitieron un informe a comienzos del año 2005, pero éste no fue hecho público a pesar de las numerosas solicitudes para que dicho informe se diera a conocer (Castro, 2005; Koljatic y Silva, 2005b; Matte, 2005).

Los mismos problemas de mal funcionamiento estadístico y persistente negación de éste por parte del comité técnico de la PSU (Comité Técnico Asesor, 2005), surgieron después del segundo año de aplicación de la prueba. Asimismo, persistieron las críticas de académicos con respecto a la escasez y la baja calidad de los ítems a disposición de los postulantes para preparar las nuevas pruebas (Chumacero, 2006; Ibáñez, 2005). Sumado a lo anterior, en la segunda aplicación se denunció una filtración en la PSU de 
Ciencias (Zúñiga, 2005a; Cento, 2005), donde 36 de 54 preguntas fueron reveladas, al menos, a dos estudiantes que asistían a un preuniversitario ("Renuncia Jefe", El Mercurio, 2005, enero 21). El rector de la Universidad de Chile prontamente atribuyó el problema a una precisa "reconstrucción de la prueba", facilitada por el hecho de que se habían repetido un número de ítems del año anterior con el objeto de equiparar pruebas (Zúñiga, 2005b). La explicación entregada por el rector fue estimada como poco plausible e inverosímil por algunos (Fontecilla, 2005; Ibáñez, 2005). Finalmente, el problema llegó a los tribunales, pero el ministro de fuero se declaró incompetente (Carvajal y Zúñiga, 2005).

El incidente de la filtración de preguntas y la explicación del rector sobre la reconstrucción de la prueba debió haber sido un serio motivo de preocupación. Si las pruebas PSU contenían un número de ítems de pruebas anteriores y los preuniversitarios enviaban a sus instructores a rendir las PSU para memorizar los ítems - como fue sugerido por los funcionarios del DEMRE y otros-, los estudiantes que asistían a dichos institutos tendrían una injusta ventaja sobre aquellos que no podían solventarlos (Valdés, 2006; Zúñiga y Olivares, 2005). Este escenario resultaba plausible dado que uno de los muchos preuniversitarios que preparaban para la PSU alardeaba en su publicidad a comienzos del año 2006 que sobre el 40\% de los postulantes del país que había obtenido puntajes máximos o "nacionales" en la PSU se había matriculado en sus programas. Este porcentaje subió al 59\% el año siguiente.

En un tema donde el secretismo y las limitaciones al acceso de información prevalecían, algunos fueron tomados por sorpresa cuando el rector de la Universidad de Chile reportó en su institución un progresivo descenso en la matrícula de los alumnos provenientes de colegios públicos municipales, de un 32\% en el 2003 a un 20\% en el 2006. La tendencia a la baja coincidía con los cambios en las pruebas y el rector así lo reconoció, cuando señaló textualmente: "El perfil social de los alumnos de la [Universidad de] Chile ha ido cambiando en el tiempo... Si en el pasado más de un tercio de los estudiantes provenían de colegios municipales, hoy es sólo el 20 por ciento... el origen social se ha ido desplazando hacia arriba producto de los sistemas de selección” (Zúñiga, 2005c; "Subieron los Puntajes”, El Mercurio, 2006, abril 16).

La declaración del rector aparecía consistente con la amplificación de la brecha de desempeño entre los postulantes de colegios privados y públicos, que creció un $11 \%$ para la prueba de Lenguaje y un 15\% para matemáticas desde su primera implementación en diciembre del 2003 hasta su tercera aplicación en diciembre del 2005. 
CUADRO N ${ }^{\circ}$ 1: $\quad$ BRECHAS EXPRESADAS COMO TAMAÑO DE EFECTO (TE)* ENTRE POSTULANTES DE ESTABLECIMIENTOS MUNICIPALES Y PRIVADOS

\begin{tabular}{|c|c|c|}
\hline & Prueba de lenguaje & Prueba de matemáticas \\
\hline TE PAA (2002) & 0,98 & 1,08 \\
\hline $95 \%$ IC & $\begin{array}{l}0,97-1,00 \\
(\mathrm{~N}=110.768)\end{array}$ & $\begin{array}{l}1,07-1,09 \\
(\mathrm{~N}=110.765)\end{array}$ \\
\hline TE PSU (2003) & 0,97 & $1,01^{(\mathrm{a})}$ \\
\hline $95 \%$ IC & $\begin{array}{l}0,95-0,98 \\
(\mathrm{~N}=89.748)\end{array}$ & $\begin{array}{l}0,99-1,03 \\
(\mathrm{~N}=89.421)\end{array}$ \\
\hline TE PSU (2004) & 1,00 & 1,07 \\
\hline $95 \%$ IC & $\begin{array}{l}0,98-1,01 \\
(\mathrm{~N}=95.050)\end{array}$ & $\begin{array}{l}1,05-1,08 \\
(\mathrm{~N}=94.808)\end{array}$ \\
\hline TE PSU (2005) & 1,08 & 1,15 \\
\hline $95 \%$ IC & $\begin{array}{l}1,06-1,09 \\
(\mathrm{~N}=95.372)\end{array}$ & $\begin{array}{l}1,14-1,17 \\
(\mathrm{~N}=95.372)\end{array}$ \\
\hline TE PSU (2006) ${ }^{(b)}$ & 1,21 & 1,29 \\
\hline $95 \%$ IC & $\begin{array}{l}1,20-1,22 \\
(\mathrm{~N}=114.936)\end{array}$ & $\begin{array}{l}1,27-1,30 \\
(N=114.226)\end{array}$ \\
\hline
\end{tabular}

Fuente de datos: D.A.R.A., P. Universidad Católica de Chile.

* El tamaño de efecto es una medida que es independiente de la escala. Se define como la diferencia entre el promedio obtenido por el grupo de postulantes de la educación privada y la educación municipal, dividido por la desviación estándar de ambos grupos.

(a) Si los puntajes de la PSU hubieran sido transformados de igual forma que la PAA, la brecha habría aumentado en un 8\% (ver Anexo). Además hay que considerar que la brecha para el año 2003 puede constituir una subestimación dado el proceso de autoselección que se observó en el grupo de postulantes de la educación municipal. La autoselección disminuyó en los años sucesivos, aun cuando hasta dos años después (PSU 2005) el contingente de alumnos municipales no recuperaba el nivel de 2002.

(b) El alza en el tamaño de efecto en la promoción 2006 puede estar, en parte, influido por la entrega de becas PSU otorgadas por el Ministerio de Educación que se tradujo en que el contingente de alumnos de colegios municipalizados aumentara. Sin embargo, la tendencia observada es consistente con el aumento de la brecha observado al año 2005. Aun si se eliminan aproximadamente 10.000 postulantes de colegios municipales con puntajes de notas de enseñanza media inferiores a 415 puntos, la brecha sigue siendo más alta que la del año anterior (PSU lenguaje y comunicación $=1,14$; PSU matemáticas $=1,21$ ).

Una explicación posible para el aumento de la brecha parecía ser la progresiva inclusión de contenidos avanzados en las pruebas. La incorporación anual de nuevos contenidos podía estar cobrando víctimas entre los postulantes del sistema municipal, puesto que éste estaba atrasado en la implementación de la jornada escolar completa (Zúñiga, 2006). Así, los alumnos de colegios municipales estaban compitiendo en términos desiguales con los postulantes de colegios privados, los que no sólo tenían jornada 
completa sino que también tenían acceso a caros programas de preuniversitario. Con el cambio se crearon nuevos nichos de mercado para los preuniversitarios cuando colegios privados contrataron programas especiales para entrenar a sus estudiantes incorporándolos dentro de las horas curriculares de la enseñanza media ("Las Lecciones”, en "En Familia, Noticias Centro de Padres”, 2005, junio). Antes de la introducción de las nuevas pruebas, quienes contrataban los servicios de preuniversitarios eran mayoritariamente estudiantes individuales. Se cumplió el pronóstico de los analistas educacionales que anticipaban que las nuevas pruebas darían una ventaja a las minorías acomodadas que podían costear un programa de entrenamiento por la vía de preuniversitarios (Beyer, 2002; Fontaine, 2002).

No obstante lo anterior, los responsables de las políticas públicas en educación no dieron signos de que las nuevas pruebas serían revisadas o de que se tomarían medidas paliativas para neutralizar los efectos adversos sobre la equidad. Más aun, los líderes del proyecto SIES continuaron afirmando sobre la base de comparaciones equívocas que no tomaban en consideración la distinta transformación de puntajes y varianza de las pruebas en el tiempo, que la PSU habría acortado la brecha entre los postulantes de colegios municipales y privados, cuando en realidad la habían acrecentado (Beyer, 2007).

\section{Discusión}

El cambio en las pruebas de admisión en Chile fue defendido empleando argumentos de que su uso mejoraría la equidad en el acceso a la educación superior, aunque ello fuera negado posteriormente por quienes lideraron el cambio de las pruebas (Miranda y Rodríguez, 2007). En lugar de sustituir la PAA por un conjunto de pruebas que medía el 100\% del contenido curricular, se podría haber definido un subconjunto de contenidos relevantes para el éxito académico (Jensen, 2000). Desde una perspectiva de selección universitaria, la inclusión de la totalidad de los contenidos fue innecesaria e imprudente, particularmente cuando la mayoría de los colegios públicos no estaba funcionando con régimen de jornada escolar completa. Con ello se violó el derecho de estos alumnos a una justa evaluación, puesto que no se cumplió con un estándar básico: "proveer a los estudiantes con el currículo y la instrucción que les permitiera la oportunidad de aprender los contenidos y habilidades medidas en las pruebas" (AERA, APA y NCME, 1999, p. 141). 


\subsection{Errores de implementación}

El proceso de cambio de pruebas estuvo plagado de improvisaciones y problemas técnicos en su implementación. Una materia especialmente inquietante fue el aumento de las brechas en los puntajes de las pruebas entre postulantes de la educación pública y privada, asociado a los cambios en el sistema de admisión. Este resultado aparece consistente con hallazgos internacionales de que la adopción de exámenes altamente competitivos no contribuye a la equidad de acceso a la educación superior en países en desarrollo y, frecuentemente, los resultados de tales cambios terminan por restringir dicho acceso (Lewis y Dundar, 2002). En un país como Chile, donde la movilidad social está estrechamente asociada con la educación universitaria recibida, la creciente evidencia de inequidad no debió haber sido ignorada por los responsables, particularmente porque el cambio en las pruebas fue presentado al público como un avance en materia de equidad.

Contrariamente a las expectativas de que las políticas educacionales serían elaboradas con criterios técnicos y atendiendo a la evidencia, las nuevas pruebas fueron implementadas sin un marco de validación adecuado. En la omisión de un marco de validación hubo responsabilidad conjunta de las autoridades que promovieron el cambio y de quienes desarrollaron las pruebas mediante el proyecto FONDEF, el cual ni siquiera mencionaba la necesidad de conducir estudios de validez antes de eliminar la batería de pruebas de admisión en uso (Bravo et al., 2000). Cuando el tema fue puesto en el tapete, en vez de corregir el déficit se le restó importancia. Luego de que académicos independientes entregaron evidencia técnica de la débil calidad de las nuevas pruebas SIES en desarrollo, se desechó el nombre y se hicieron concesiones menores para calmar la conmoción pública. Dichas concesiones no resolvieron el problema central, es decir, la falta de evidencia para garantizar la calidad de las nuevas pruebas de admisión y asegurar una transición fluida entre ambos sistemas, evitando así consecuencias negativas para los postulantes.

El cambio de las pruebas fue impuesto por las autoridades políticas y no hubo transparencia con respecto a su verdadero propósito, cual era garantizar la sustentabilidad de la reforma de educación media y evaluar sus resultados. Si las pruebas no fueron instrumentos concebidos y desarrollados para evaluar la enseñanza media, resulta ilógico que los autores del cambio hayan insistido en incorporar el $100 \%$ de los contenidos cubiertos en las asignaturas en la enseñanza media en lugar de restringir éstos a aquellos relevantes para el buen desempeño en la educación superior. Por 
otro lado, el intento de transformar las pruebas de admisión en una evaluación de la enseñanza secundaria fue un error técnico, simplemente porque los exámenes de admisión y de egreso de la enseñanza media sirven a diferentes propósitos y requieren diferentes tipos de instrumentos. Como lo expresó un destacado experto internacional en medición": "Si una prueba midiera el currículum enseñado y los estudiantes aprendieran lo esperado, la distribución del puntaje mostraría una asimetría negativa. Esto sería esperado y deseable. Sin embargo, para las pruebas de admisión lo deseable es tener una amplia dispersión de los puntajes para maximizar su confiabilidad y validez para predecir éxito... por consiguiente, se esperaría y sería deseable una distribución de puntajes simétrica y en forma de campana. Es improbable, por decir lo menos, que los dos propósitos — selección universitaria y evaluación de resultados de enseñanza media — pudieran ser bien logrados por medio de un mismo instrumento". Pero incluso si tal instrumento mágico pudiera ser concebido para el doble propósito de selección y evaluación de resultados de la enseñanza media, debió haberse recogido evidencia de validez para ambos objetivos en relación al contenido de las pruebas, procesos de respuesta, estructura interna, relaciones con otras variables y las consecuencias del uso de las nuevas pruebas, como medio para evaluar su calidad técnica (AERA, NCME y APA, 1999).

Aun para los planes mejor diseñados por los más reconocidos expertos en políticas públicas, algunas consecuencias no anticipadas parecen ser inevitables (Kingdon, 1995). Sin embargo, en este caso las probables consecuencias negativas para la equidad fueron señaladas a las autoridades y los responsables del proyecto, pero inexplicablemente ellos optaron por ignorar los riesgos implicados para los postulantes de colegios municipales. Pasar por alto la evaluación de las consecuencias negativas fue el resultado de un proceso que se caracterizó por una planificación inadecuada y una defectuosa implementación, junto a la gestación de expectativas no satisfechas en relación a la potencialidad de las nuevas pruebas para promover un equitativo acceso a la educación superior. El resultado de un aumento en la inequidad asociado al proceso de cambio traicionó la esencia misma de la reforma educacional de los 90 que buscaba lo contrario, y mostró que una cosa es implementar una política y otra demostrar que es efectiva (Odden, 1991). En este sentido, la experiencia chilena podría ser caracterizada como

${ }^{5}$ Ronald Hambleton, profesor de la Universidad de Massachussetts en Amherst, EE.UU., es autor de ocho libros de medición educacional y más de 600 artículos y reportes especializados en el tema. Fue Presidente del National Council for Measurement in Education y es editor de las más prestigiosas revistas internacionales de medición. 
un caso emblemático de una deficiente implementación de políticas, donde el desarrollo de las pruebas fue realizado de una manera desprolija y estuvo asociado a serias consecuencias negativas (Heyneman, 1987).

\subsection{Transparencia y rendición de cuentas}

Los expertos concuerdan en que una entidad examinadora está en mejor posición para hacer un buen trabajo en lo técnico cuando la agencia es independiente del financiamiento gubernamental y del control político (Heyneman, 1987). Éste no fue el caso en Chile donde el DEMRE era un departamento dentro de la Universidad de Chile, institución que dependía de fondos de gobierno. El cambio de las pruebas fue planificado e implementado centralmente en el Ministerio, y el personal técnico del DEMRE que terminó haciéndose cargo de desarrollar y aplicar las nuevas pruebas no estaba en posición de demandar las condiciones necesarias para hacer un trabajo riguroso. Si esta institución hubiese sido autónoma, habría existido la posibilidad de que la calidad de las pruebas y su impacto en la equidad hubiesen sido adecuadamente abordados. Aun cuando existió un comité técnico para la PSU nombrado por el Consejo de Rectores para supervisar el proceso, algunos de sus miembros no eran independientes. Aquellos que planearon e implementaron el cambio terminaron actuando como los únicos evaluadores de la calidad de la PSU (Repetto, 2006; Koljatic y Silva, 2005a). Sus informes de las nuevas pruebas se ajustaban a un tipo de pseudo-evaluación que se puede caracterizar como "estudios inspirados en las relaciones públicas, basados en una necesidad de [proveer] información propagandística, que buscan construir una imagen positiva de una política o programa" (Stufflebeam y Webster, citados en Brown y Wildawsky, 1983, p. 189). Sin auditorías independientes, la opinión pública sólo recibía información oficial del comité asesor del Consejo de Rectores, algunos de cuyos reportes adolecían de evidentes limitaciones con el agravante de que investigadores educacionales externos no tenían acceso a las bases de datos correspondientes (Arellano, 2006; Repetto, 2006; Koljatic y Silva, 2006; Beyer, 2007). Frente a esta situación, la Cámara de Diputados de Chile elevó una solicitud para gestar transparencia, que incluía "la apertura completa de las bases de datos y de toda la información técnica existente sobre la PSU para que quede a disposición de la comunidad académica y de quien lo solicite” y la elaboración de bases para una licitación internacional destinada a contratar los servicios de una institución independiente, del más alto nivel, para auditar las pruebas en 
forma integral y periódica (“Piden Licitación Internacional”, en "Noticias Cámara de Diputados”, 2007, enero 24)

La falta de transparencia y el ocultamiento de información que marcó el proceso de cambio quedaron en evidencia con la negativa de hacer público el informe del ETS sobre la PSU. Esto dejó en claro la necesidad de abogar por una agencia autónoma a cargo del desarrollo de las pruebas de admisión, una organización animada por estándares profesionales y que no sucumbiera a presiones políticas, tal cual fuera sugerido por Heyneman y Ransom (1990).

Aunque en otras partes del mundo pueden encontrarse casos documentados de falta de atención a temas de validez que resultan en usos inapropiados de pruebas educacionales (Hartmann y Fisher, 1999; Popham, 2003), en los países desarrollados hay mayor conciencia por parte de las autoridades responsables de las políticas públicas y por quienes desarrollan las pruebas de la importancia de los estudios de validez para promover el uso sensato y ético de este tipo de pruebas. Suele darse también una mayor apertura a reconocer los errores y corregirlos. En el caso de EE. UU., ello puede deberse a que su sistema judicial regula y estimula las buenas prácticas de medición promovidas por la NCME, AERA y APA (1999). En tal sentido, los consultores de proyectos internacionales podrían contribuir a promover las buenas prácticas en países en desarrollo abogando por que las pruebas sean desarrolladas conforme a los mismos altos niveles que están obligados a respetar en sus propios países para proteger los derechos de quienes son evaluados.

\subsection{Sugerencias para gestar un sistema de pruebas de admisión transparente y de calidad en Chile}

Para gestar un sistema transparente el DEMRE — $\mathrm{u}$ otra entidad independiente- debe constituirse como una organización autónoma, sin fines de lucro y enfocada en desarrollar un trabajo técnico de calidad. La entidad debe tener autonomía en su manejo presupuestario y estar obligada a reinvertir sus excedentes en mejorar las pruebas, así como a responder ante la comunidad académica y ante la opinión pública por la calidad de su trabajo.

Un primer paso hacia la transparencia es dar a conocer el informe completo de la auditoría del Educational Testing Service (ETS) realizada a la PSU. El país no tiene claro cuáles fueron los términos ni los alcances de esta auditoría contratada por la Universidad de Chile. Existen preguntas que no 
deben ser dejadas de lado, tales como si la PSU mide lo que debe medir y cuáles han sido las externalidades del cambio, particularmente en relación a la equidad de acceso. El informe de la auditoría debe hacerse público y su discusión, con participación de quienes lo emitieron (expertos internacionales del ETS) y otros especialistas extranjeros calificados e independientes, puede ser un camino para abrir el diálogo y dar transparencia en esta materia de tanta relevancia para el país. No es aceptable que quienes diseñan e implementan un cambio sean quienes tengan la última palabra para evaluar la calidad del trabajo realizado. El Consejo de Rectores debería invitar a formar parte de su comité estable a reconocidos expertos mundiales en el desarrollo de pruebas. Los costos de las auditorías y de un comité independiente y de alto nivel se deberían financiar con los excedentes que genera la administración del sistema de admisión.

En este sentido, conviene mirar la experiencia de países que cuentan con sistemas de admisión universitaria de calidad como Suecia, donde se ha conformado un directorio responsable del proceso, que es independiente y donde se hacen auditorías técnicas periódicas, y desde luego dadas a conocer a la opinión pública. El acceso a las bases de datos por parte de investigadores independientes se considera un derecho, a diferencia de lo que ocurre en Chile donde impera el secretismo y monopolio de la información. Asimismo, la entrega de los facsímiles aplicados el año anterior es indispensable para garantizar que todos los postulantes estén en igualdad de condiciones.

Finalmente, es de esperar que esta oscura historia del cambio de pruebas y el daño ocasionado a estas cuatro generaciones de estudiantes provenientes del sector municipalizado sirvan para revisar lo que se está haciendo en la actualidad y que las nuevas autoridades se propongan articular un sistema de admisión transparente y de calidad. 
ANEXO

Comparación de Puntajes PAA y PSU para algunos percentiles seleccionados en la Prueba de Matemáticas

\begin{tabular}{lccl}
\hline & $\begin{array}{c}\text { Puntaje con } \\
\text { transformación } \\
\text { PAA }\end{array}$ & $\begin{array}{c}\text { Puntaje con } \\
\text { transformación } \\
\text { PSU }\end{array}$ & $\begin{array}{c}\text { Diferencia } \\
\text { PSU-PAA }\end{array}$ \\
\hline Percentil 5 & 312 & 310 & -2 \\
Percentil 10 & 329 & 356 & +17 \\
Percentil 15 & 343 & 388 & +45 \\
Percentil 20 & 359 & 410 & +41 \\
Percentil 25 & 374 & 423 & +49 \\
Percentil 30 & 392 & 440 & +48 \\
Percentil 35 & 409 & 456 & +47 \\
Percentil 40 & 429 & 467 & +38 \\
Percentil 45 & 452 & 485 & +33 \\
Percentil 50 & 475 & 499 & +24 \\
Percentil 55 & 499 & 512 & +13 \\
Percentil 60 & 524 & 525 & +1 \\
Percentil 65 & 550 & 540 & -22 \\
Percentil 70 & 576 & 554 & -30 \\
Percentil 75 & 604 & 570 & -46 \\
Percentil 80 & 634 & 588 & -60 \\
Percentil 85 & 667 & 607 & -72 \\
Percentil 90 & 705 & 633 & -84 \\
Percentil 95 & 751 & 667 & -84 \\
Percentil 96 & 763 & 679 & -66 \\
Percentil 97 & 776 & 710 & -30 \\
Percentil 98 & 786 & 739 & \\
Percentil 99 & 805 & & \\
\hline
\end{tabular}

Fuentes: Del Pino y Aravena 2004 (columnas 1 y 2). Columna de diferencias entre PSU y PAA y nota explicativa de la tabla, de los autores.

Como se puede observar en esta tabla, la aparente reducción de la brecha en la primera aplicación de la PSU se debió a la diferencia en la transformación de puntajes entre la PAA y la PSU. A quienes obtenían puntajes bajos, la transformación de puntajes de la PSU les favoreció. Algunos "ganaron" hasta 49 puntos (ver percentil 25). En cambio, los alumnos que sacaban puntajes altos la transformación los perjudicó hasta en 84 puntos (ver percentil 95). Dicho de otra forma, la transformación PSU "subió” la cola inferior de la distribución (mayormente representada por postulantes de colegios municipalizados) y "castigó” la cola superior de la distribución, (mayormente representados los colegios particulares). La aparente reduccion de la brecha fue consecuencia de la transformación empleada, como señaló Beyer (2004). 


\section{REFERENCIAS}

American Educational Research Association (AERA), American Psychological Association (APA) and National Council on Measurement in Education (NCME) (1999): Standards for Educational and Psychological Testing. Washington, DC.: AERA, APA y NCME.

Aravena, N. y P. Molina (2002, mayo 20): "El Límite a la Autonomía: Las Platas Fiscales”. En El Mercurio de Santiago, p. C3.

Arellano, S. (2006, diciembre 10). "La PSU No Pasa la Prueba”. En El Mercurio de Santiago, p. B2.

Astorga, L. (2002, junio 30): “Duda Maestra”. En El Mercurio de Santiago, p. D25.

Atkinson, R. (2001): “Achievement Versus Aptitude in College Admissions”. En Issues in Science and Technology, Vol. 18 № 2, pp. 31-36.

Beyer, H. (2000): “Educación y Desigualdad de Ingresos: Una Nueva Mirada”. En Estudios Públicos, № 77 (verano), pp. 97-130.

(2002, abril 25): “Sobre las Pruebas de Ingreso a las Universidades”. En El Mercurio de Santiago, p. A2.

- (2004, enero 18): "Ilusiones Estadísticas de la PSU”. En El Mercurio de Santiago, p. A2.

(2007, enero 17): “Equidad en la PSU”. En El Mercurio de Santiago, p. A2.

Bortolaso, J. (2002, junio 17): “SIES: Dudas y Certeza”. En El Mercurio de Santiago, p. A2.

Bravo, D., J. Manzi, O. Larrañaga, D. Contreras, E. Himmel, R. Rosas y M. Sevilla (2000): "Reformulación de las Pruebas de Selección a la Educación Superior". Octavo Concurso Nacional de Proyectos de Investigación y Desarrollo, FONDEF-CONICYT, Santiago, Chile.

Bravo, D. y J. Manzi (2002, abril 28): "Equidad y Resultados". En El Mercurio de Santiago, p. E6-E7.

Browne, A. y A. Wildawsky (1983): What Should Evaluation Mean to Implementation”. En J. Pressman y A. Wildawsky (eds.), Implementation. Berkeley: University of California Press.

Brunner, J. (2002, junio 19): "SIES: Tres Preguntas y la Responsabilidad de las Universidades”. En La Segunda, Santiago, p. 7.

(2005): "Comparative Research and Public Policy: From Authoritarianism to Democracy”. En Peabody Journal of Education, Vol. 80 No 1, pp. 100-106.

Bustos, E., N. Galanti y H. Díaz (2002, abril 10): "Proyecto SIES”. En El Mercurio de Santiago, p. A2.

Carvajal, C. y V. Zúñiga (2005, enero 27): “El Ministro de Fuero Se Declara Incompetente en el Caso PSU”. En El Mercurio de Santiago, p. C8.

Castro, R. (2005, diciembre 10): “Evaluación de PSU”. En El Mercurio de Santiago, p. A2.

Cento, C. (2005, enero 14): "Lily Pérez a Riveros: Rector Se Equivocó de Blanco”. En La Segunda, Santiago, p. 7.

Chakwera, E., D. Khembo, y S. Sireci (2004, junio 28): "High-Stakes Testing in the Warm Heart of Africa: The Challenges and Successes of the Malawi National Examinations Board”. En Educational Policy Analysis Archives, Vol. 12, № 29. En http://epaa.asu.edu/epaa/v12n29 
Chumacero, R. (2006): “De Vuelta a Clases”. En Estudios Públicos, 103 (invierno), pp. 278-306.

Comisión de Matemática (CEP) (2002): “El SIES y la Matemática”. En Estudios Públicos, № 87.

Comisión Nuevo Curriculum de la Enseñanza Media y Pruebas del Sistema de Admisión a la Educación Superior (2000, noviembre 22): "Informe Sometido en Consulta Previa a la Ministra de Educación”.

Comité Técnico Asesor, Honorable Consejo de Rectores de las Universidades Chilenas (2004): "Resultados de la Aplicación de Pruebas de Selección Universitaria Admisión 2004”. Santiago.

(2005): "Resultados de la Aplicación de Pruebas de Selección Universitaria Admisión 2005”. Santiago.

Delannoy, F. (2000): "Education Reforms in Chile, 1980-98: A Lesson in Pragmatism”. En World Bank (ed.), Country Studies Education Reform and Management Publication Series. Washington D.C.: The World Bank.

Del Pino, G. y R. Aravena (2004): “Análisis Estadístico de los Efectos de la Normalización de Puntajes en las Pruebas de Selección Universitaria”. En Statistica et Societatis, No 3, pp. 1-15.

Delpiano, G. (2004, mayo): "PSU”. En FEUC al Debate, p. 3. Santiago, Chile: Informativo de la Federación de Estudiantes de la U.C.

Díaz, E., E. Himmel y S. Maltes (1990): “Evolución Histórica del Sistema de Selección a las Universidades Chilenas 1967-1989”. En M. J. Lemaitre (ed.), La Educación Superior en Chile: Un Sistema en Transición. Santiago: CPU, pp. 305-358.

Dusaillant, F. (2002): "Debate Público: Comportamiento Estratégico y Respuestas Graduadas en el SIES”. En Estudios Públicos, No 87, pp. 345-368.

EducarChile.cl (2002, abril 26): "SIES: Un Desafío para la Enseñanza Media”. En http:// www.educarchile.cl/Portal.base/Web/VerContenido.aspx?ID=73956\&GUID= 733b5d97-4365-4675-afbe-aa45878b68de 8tomado el 22 de mayo de 2002).

El Diario Austral, Valdivia (2002, julio 21, p. A12): “El SIES Comenzará a Ser Aplicado a Contar del 2003".

El Mercurio de Santiago (2002, julio 4, p. C11): “Colegio de Profesores Manifestó su Apoyo al SIES".

(2003a, marzo 28, p. C1): “Académicos Detectan Recelos hacia Prueba”. (2003b, abril 17, p. A1): "Postulantes en Desventaja con la Nueva PAA". Suplemento Alternativas Académicas (2003c, julio 8, pp. 12-13): "El Ajuste para la PSU”.

- Suplemento Alternativas Académicas (2003d, julio 8): "Pruebas de Selección a la Educación Superior”. En http://www.alternativas.mercurio.cl/secciones/003/ 200307080012.html (tomado el 14 de julio de 2003).

(2003e, septiembre 27, p. C1): “En Noviembre Habrá Ensayo de la PSU”. (2003f, octubre 7, p. A1): "Universidades dan más tiempo a 3 tests de la PSU”. (2005, enero 21, p. A1): "Renuncia Jefe de la PSU por el Caso Filtración”. (2006, abril 16, pp. E2-E4): "Subieron los Puntajes, pero Bajó la Diversidad Social”.

El Mostrador.cl. (2002, abril 4): "Dan a Conocer Detalles de Examen que Reemplazará a la PAA”. 
Elmes, L., M. Cáceres, H. Steil, J. Aragoneses, L. Budge, M. Góngora, et al. (2002): "Prueba de Lenguaje: Críticas y Propuestas". En Estudios Públicos, N ${ }^{\circ} 88$ (primavera) pp. 265-350.

“En Familia: Noticias del Centro de Padres Villa María Academy” (2005, junio): “Las Lecciones del Simce y la PSU”. Santiago, Chile.

Eyzaguirre, B. y C. Le Foulon (2002, junio 9): “SIES: Un Proyecto Prematuro”. En El Mercurio de Santiago, cuerpo Artes y Letras, pp. E8-E9.

Fermandois, J., D. Ahumada, F. Arellano, R. Céspedes, A. Parraguez, C. Pérez et al. (2002): "La Evaluación del Conocimiento Histórico". En Estudios Públicos $\mathrm{N}^{\circ} 88$ (primavera), pp. 427-454.

Fontaine, A. (2002, mayo 12): "Peligro en el SIES”. En El Mercurio de Santiago, “Artes y Letras”, p. E1.

Fontecilla, J. (2005, enero 27): “Filtración PSU”. En El Mercurio de Santiago, p. A2.

Friedman, E., L. Arancibia, R. Bamon, F. Dusaillant, R. Rebolledo, R. Rodríguez et al. (2002): “El SIES y la Matemática”. En Estudios Públicos, No 87 (invierno), pp. 369-395.

García-Huidobro, B. (2002, junio 9): "Prueba SIES: Experiencia Personal”. En El Mercurio de Santiago, p. A2.

Geisser, S. y R. Studley (2001): "UC and the SAT: Predictive Validity and Differential Impact of the SAT 1 and SAT 2 at the University of California”. Document of the Office of the President, University of California.

Hartmann, C. E. y G. L. Fisher (1999): "When Good Ideas Become Bad Policy: Implications of the National Voluntary Mathematics Test”. En Education Policy, Vol. 13, No 3, pp. 371-386.

Hawes, G. (2004, julio 13): "Pruebas de Selección Universitaria”. En El Mercurio de Santiago, p. A2.

Henríquez, J. (2003, agosto 6): "Me Impresiona que Estemos Haciendo el Mismo Cambio en EE.UU. y Chile”. En La Segunda, Santiago, p. 9.

Henrysson, S. (1971): “Gathering, Analyzing, and Using Data on Test Items”. En R. L. Thorndike (ed.), Educational Measurement, 2da edición. Washington, DC: American Council on Education.

Herrera, M. (2001, agosto 5): “Desencuentros Curriculares”. En El Mercurio de Santiago, pp. D15-D16.

Heyneman, S. (1987): "Uses of Examinations in Developing Countries: Selection, Research and Education Sector Management”. En International Journal of Educational Development, Vol. 7, No 4, pp. 251-263.

(2003): “Misconduct in Education”. En J. Guthrie (ed.), Encyclopedia of Education. New York: Mac Millan, Vol. 5, pp. 1659-1668.

Heyneman, S. y A. Ransom (1990): "Using Examinations and Testing to Improve Educational Quality”. En Educational Policy, Vol. 4, No 3, pp. 177-192.

Hojman, S., A. Barriga, C. Barros, V. Cifuentes, R. Contreras, I. Fredes et al. (2002): “Prueba de Ciencias: Críticas y Propuestas”. En Estudios Públicos, N ${ }^{\circ} 88$ (primavera), pp. 251-426.

Ibáñez, D. (2002, junio 28): “La Prueba SIES y la UTI”. En El Mercurio de Santiago, p. A2.

- (2005, marzo 31): “PSU: ¿Aspectos Colaterales?”. En El Mercurio de Santiago, p. A2. 
(2005, mayo 29): “Joyitas de la PSU”. En El Mercurio de Santiago, p. A2.

Jensen, A. R. (2000): “The Dilemma of Group Differences”. En Psychology, Public Policy, and Law, Vol. 6, No 1, pp. 121-127.

Jalilie, J. (2002, junio 13): “Denuncian Campaña Anti-SIES”. En La Nación, Santiago, p. 10.

Kingdon, J. (1995): Agenda Alternatives and Public Policies. N.Y.: Harper Collins.

Koljatic, M. (2004, agosto 27): “PSU: Problemas Actuales y Desafíos Futuros”. Manuscrito, Universidad Andrés Bello, Santiago, Chile.

Koljatic, M. y M. Silva (2002): "Del SIES al PAT: Un Arreglo 'a la Chilena'”. En Administración y Economía UC, No 48, pp. 21-23.

(2005a, noviembre 27): “Las ‘Verdades Oficiales’ de la PSU”. En El Mercurio de Santiago, pp. E14-E15.

(2005b, diciembre 22): "Resultados de la PSU”, El Mercurio de Santiago, p. A2. (2006): "Validación de la PSU: Comentarios al 'Estudio Acerca de la Validez Predictiva de los Factores de Selección a las Universidades del Consejo de Rectores'”. En Estudios Públicos No 104 (primavera), pp. 331-346.

La Segunda, Santiago (2000, noviembre 24, p. 12): "Consejo de Rectores Decide en Diciembre Cambios Finales a la PAA".

(2002a, julio 4, p. 11): “Comisión de la U. de Chile Propone Postergar la Aplicación de la Prueba SIES”.

- (2002b, julio 4, p. 8): "SIES la Presión del Cronograma”. (2002c, julio 17, p. 39): "Riveros Propone Aplicación Paralela de SIES y

PAA".

- (2002d, junio 19, p. 5): "U. de Chile Pone Cautela a Prueba SIES”.

(2002f, julio 22, p. 5): “Comisión de Expertos del CEP 'Demuele’ la Prueba SIES de Matemáticas”.

(2002g, julio 25, p. 43): "Rectores de 25 Universidades Tradicionales Debaten el Futuro de la prueba SIES”.

(2002h, julio 30, p. 4): "Rectores Crean Consejo Asesor para Respaldar al SIES".

(2002i, agosto 16, p. 62): “Académicos de la USACH Preparan Crítico Documento sobre el Peligro del SIES”.

(2002j, agosto 26, p. 2): "Guerra Declarada entre Ministra Aylwin y Rector de la U. de Chile por el SIES”.

(2002k, agosto 29, p. 4): "Ni la PAA ni el SIES".

(2002l, agosto 30, p. 10): “Transacción y Transición entre PAA y SIES”.

(2003a, enero 23, p. 4): "Postulantes Tendrán Menos Meses para Ejercitar con Facsímiles de la Nueva PAA”.

(2003b, abril 16, p. 2): "Escasez de Facsímiles: Expertos Advierten Desventajas para los que Darán Nueva PAA”.

(2003c, julio 30, p. 10): "Puntajes de los Nuevos Exámenes Serán Muy Parecidos a los de la PAA”.

- (2003d, noviembre 2, p. 103): “'Padres’ de la PSU Enuncian sus Aspectos Clave".

- (2003e, noviembre 28, p. 3): “Alumnos Lograron Mejores Puntajes en la PSU Comparado con la PAA". 
(2004a, enero 12, p. 2): "Resultados de la PSU Castigan a Colegios Particulares y Suben los Municipales”.

(2004b, enero 23, p.2): "UES Tradicionales No Logran Llenar Vacantes”.

Labarca, R. (2002, abril 12): "Al SIMCE lo que Es del SIMCE, y a la PAA lo que Es de la PAA”. En El Mostrador.cl

Las Ultimas Noticias, Santiago, Chile (2002a, junio 13): “Ministerio de Educación Reconoció Problemas de la Prueba SIES”. En http://www.lun.com/NoticiasDia/ tipo2.asp?idnoticia=C374208447916667 (tomado en abril 2003).

(2002b, junio 13): “Ministra Aylwin Valida Proyecto SIES”. En http:// www.lunc.cl/modulos/busqueda/searchleft_canales_new.asp?idnoticia= C374207660300926 (tomado el 27 de septiembre de 2003).

Lewis, D. y H. Dundar (2002): "Equity Effects of Higher Education in Developing Countries: Access, Choice, and Persistence”. En D. Chapman y A. E. Austin (eds.), Higher Education in the Developing World: Changing Contexts and Institutional Responses. Westport, CT: Greenwood Press, pp. 169-194.

Manzi, J. y D. Bravo (2002, mayo 19): "SIES: Los Peligros de lo que No Es”. En El Mercurio de Santiago, pp. E6-E7.

Matte, A. (2005, noviembre 11): "Validación de la PSU”. En El Mercurio de Santiago, p. A2.

Merriam, S. (1998): Qualitative Research and Case Study Applications in Education. San Francisco: Jossey-Bass.

Miranda, M. y Rodríguez, C. (2007, enero 14): "La PSU Refleja la Inequidad de la Educación, Pero No la Provoca”. En El Mercurio de Santiago, p. A29.

Muñoz, A. (2002, junio 23): "Experto Afirma que la PAA Es Antigua y Tiene Preguntas Pobres”. En La Tercera, Santiago, p. 15.

Navarro, S. (2002, abril 12): “Proyecto SIES”. En El Mercurio de Santiago, p. A2.

Noticias Cámara de Diputados (2007, enero 24). Piden Licitación Internacional para Auditar PSU.

Odden, A. (1991): "New Patterns of Education Policy Implementation and Challenges for the 1990s". En A. Odden (ed.), Education Policy Implementation. Albany, N.Y.: State University of New York Press.

Parada, R. (2002, agosto 23): “Proyecto SIES e Investigación”. En Diario El Sur, Concepción, Chile, p. 3.

Popham, J. (2003): “Seeking Redemption for Our Psychometric Sins”. En Educational Measurement: Issues and Practice, Vol. 22, № 1, pp. 45-48.

Repetto, A. (2006, diciembre 23): “La Evaluación de la PSU”. En La Tercera, Santiago, p. 34.

Rodríguez, R. (2002, abril 4): "Prueba de Matemática”. En El Mercurio de Santiago, p. A2.

Rosso, P. (2002a, julio): “La Universidad Católica y el SIES”. En Visión Universitaria, $\mathrm{N}^{\circ}$ XVI, Pontificia Universidad Católica de Chile.

(2002b, agosto): “Carta a los Miembros de la Comunidad Universitaria”, Pontificia Universidad Católica de Chile.

Sepúlveda, C. (2002, julio 29): “El SIES Es un Proyecto en Desarrollo”. En El Mercurio de Santiago, p. A2.

Silva, M. \& Koljatic, M. (1999, enero 26): “¿Qué Hacer con la PAA?” En El Mercurio de Santiago. 
The World Bank (2001, octubre 19): “Implementation Completion Report. (CPL-38830; SCL 38836) on a Loan in the Amount of US\$35 Million to the Republic of Chile for a CL-Secondary Education”. Latin America and the Caribbean Regional Office. Washington D.C.

Urzúa, R. (2002a, agosto 23): “La U y la USACH Tomarán PAA en Admisión 2003”. En Las Ultimas Noticias, Santiago, p. 6.

(2002b, julio 10): “Expertas Se Enfrentan por Prueba SIES”. En Las Ultimas Noticias, Santiago, p. 6.

Valdés, S. (2006, enero 23): “Cómo Entrar a Medicina”. En El Mercurio de Santiago, p. A2.

Valenzuela, E. y C. Cousiño (2002, junio 12): “Las Desventuras de una Pregunta SIES”. En El Mercurio de Santiago, p. C7.

Vial, G. (2001, agosto 14): “Alumnos y Establecimientos frente al SIES”. En La Segunda, Santiago, p. 9.

"Visión Universitaria, Pontificia Universidad Católica de Chile” (2002, julio): “Seminario Prueba de Admisión a las Universidades Chilenas".

Zúñiga, V. (2005a, enero 12): "Universidad de Chile Investiga Presunta Filtración en la PSU”. En El Mercurio de Santiago, p. C7.

(2005b, enero 24): “Evitarán Efecto de 'Reconstrucción' de PSU”. En El Mercurio de Santiago, p. C7.

(2005c, agosto 20): “Cambia Perfil Estudiantil de la U. de Chile”. En El Mercurio de Santiago, p. C12.

(2006, enero 23): “Los Rectores Dan el Sí pero Condicionado”. En El Mercurio de Santiago, p. C7.

Zúñiga, V. y R. Olivares (2005, enero 21): “Funcionarios Declaran por el Lío de la PSU”. En El Mercurio de Santiago, p. C9. 